\title{
Optimal Capacitor Placement for Loss Reduction in Distribution Systems Using Bat Algorithm
}

\author{
Mrs. V. Usha Reddy ${ }^{1}$ A. Manoj ${ }^{2}$ \\ ${ }^{1}$ Assistant Professor, Department of EEE, SVU College of Engineering, Tirupati, Andhra Pradesh, India. \\ ${ }^{2}$ Department of EEE, SVU College of Engineering, Tirupati, Andhra Pradesh, India
}

\begin{abstract}
Electricity, today, has not only become a necessity but also a tool for determining the economic standing and growth of a nation. The exponential growth in demand over the past two decades and the widening gap between demand and supply is a growing concern. So as to reduce this gap, in addition to adding new generating units, automation technology is being employed for reducing the $T \& D$ losses and therefore the increasing necessity of fast and efficient algorithms. This paper presents a two stage approach: first, Fuzzy Logic is used to find optimal capacitor locations and then Bat Algorithm is used to find optimal capacitor sizes in order to minimize losses. The proposed method is tested on 15-bus and 34-bus test systems and the results are presented.

Keywords —Bat Algorithm, Capacitor placement, Distribution system, Fuzzy logic, Metaheuristic
\end{abstract}

\section{INTRODUCTION}

New and efficient algorithms for minimising the losses in distribution systems have assumed greater significance recently since distribution automation requires the most efficient operating scenario for economic viability variations. In India, with estimated T\&D losses of $24 \%$ in 2011 and the target of reducing it to $17.1 \%$ by $2017 ; 14.1 \%$ by 2022 , the need for fast and efficient methods to reduce losses is imminent. Studies have indicated that as much as $18 \%$ of total power generated is wasted in the form of losses at the distribution level only. Capacitors are the most commonly used devices for minimizing the losses in distribution system. The usage of capacitors results in the improvement of power factor, voltage profile, power loss reduction and available capacity of feeders. With these various objectives in mind, Optimal Capacitor Placement (OCP) aims to determine capacitor location and its size.

Even though various techniques, like nonlinear optimization techniques [1-5], heuristics [6,7] and combinational optimization techniques [8], used to solve OCP problem have various merits, their efficacy relies entirely on the goodness of data. Advanced techniques like neural networks, fuzzy logic, genetic algorithms (GA) and particle swarm optimization (PSO) [9-12] overcame this problem. Two stage approach using Fuzzy and other algorithms [13-16] further improved the results.

In this paper, with a little attention given to load flow analysis of distribution network; a two stage approach to solve OCP problem is presented. First, fuzzy logic is used to determine the locations of optimal capacitors and then Bat Algorithm (BA) is used to determine size of optimal capacitors for loss reduction.

With distribution automation, many applications \& functions are coming up requiring a robust and efficient power flow solution method. The Newton-Raphson and fast decoupled load flow solution technique and a host of their derivatives have efficiently solved the Well Behaved Power Systems for a long time. Distribution networks, because of the some of the following special features, fail in the category of Ill-conditioned Power Systems:

- Radial or near radial structure

- High R/X ratios

- Multi-phase, unbalanced operation

- Unbalanced distributed load and

- Dispersed generation

Hence, special techniques for solving distribution networks which exploit their radial nature have long been identified.

A modified load-flow technique [17] is considered for solving radial distribution networks. This method involves only the evaluation of a simple algebraic expression of receiving-end voltages; takes zero initial loss for computation of voltage of each node and considers flat voltage start to incorporate voltage convergence. This method is very efficient, has good and fast convergence characteristics. 


\section{OPTIMAL CAPACITOR LOCATIONS}

The efficiency of methods prior to Fuzzy logic, even though good, depends mainly on the goodness of data. Fuzzy logic provides a remedy for any lack of uncertainty in the data. Fuzzy logic has the advantage of including heuristics and representing engineering judgments into the optimal capacitor location problem. Furthermore, the solutions obtained from a fuzzy algorithm can be quickly assessed to determine their feasibility in being implemented in the distribution system. While designing a fuzzy logic for identifying optimal capacitor locations, two objectives are considered mainly. They are:

i. $\quad$ To minimise the real power loss and

ii. To maintain voltage within permissible limits

The two inputs for Fuzzy Inference System (FIS) are per unit nodal voltages and power loss indices (PLI). The output is Capacitor Suitability Index (CSI). The FIS contains a set of rules that determine the capacitor placement suitability of each node in the distribution system.

The power loss index at each node of the distribution system can be determined by following the steps given below:

i. Perform the load flow for the original system to obtain the real and reactive power losses.

ii. After compensating the total reactive load at every node of the distribution system, perform the load flow to calculate the power loss reduction.

iii. The loss reductions are linearly normalised into a range of $[0,1]$ with largest loss reduction having a value of ' 1 ' and the smallest loss reduction having a value of ' 0 '.

iv. PLI at $\mathrm{n}^{\text {th }}$ node is now determined by using the formula given below:

$$
\operatorname{PLI}(n)=\frac{\text { lossreduction }(\mathrm{n}) \text {-lossreduction }(\min )}{\text { lossreduction }(\max )-\text { lossreduction }(\min )}
$$

The inputs and outputs of FIS are modeled by fuzzy membership functions. PLI value varies from 0 to 1. The membership functions for PLI are triangular and are denoted by L, LM, M, HM and H. The value of per unit nodal voltage ranges from 0.9 to 1.1. The membership functions for per unit nodal voltages are trapezoidal and triangular and are denoted by $\mathrm{L}, \mathrm{LN}, \mathrm{N}, \mathrm{HN}$ and $\mathrm{H}$. CSI value varies from 0 to 1 . The membership functions for CSI are triangular and are denoted by L, LM, M, HM and $\mathrm{H}$. The membership functions of the variables are shown in figures given below:

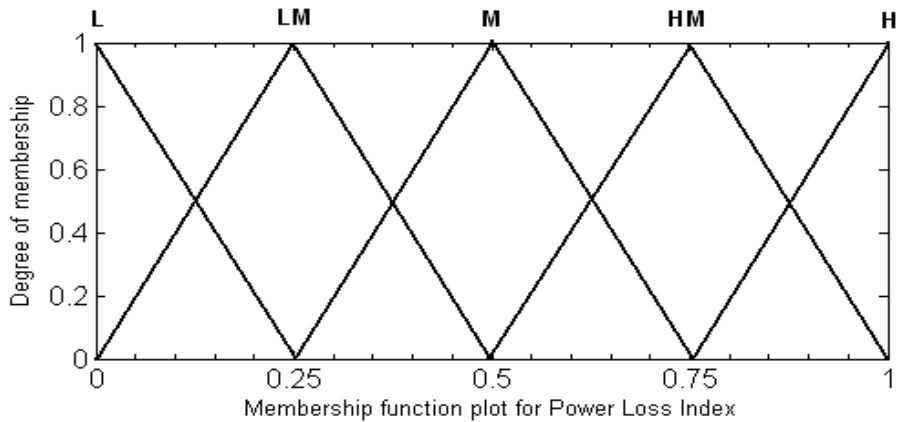

Figure 1. Membership function plot for PLI

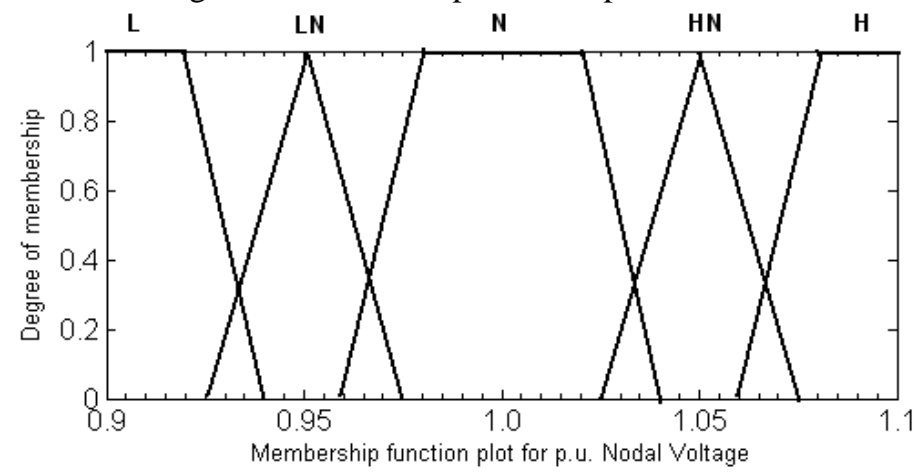

Figure 2. Membership function plot for per unit nodal voltage 


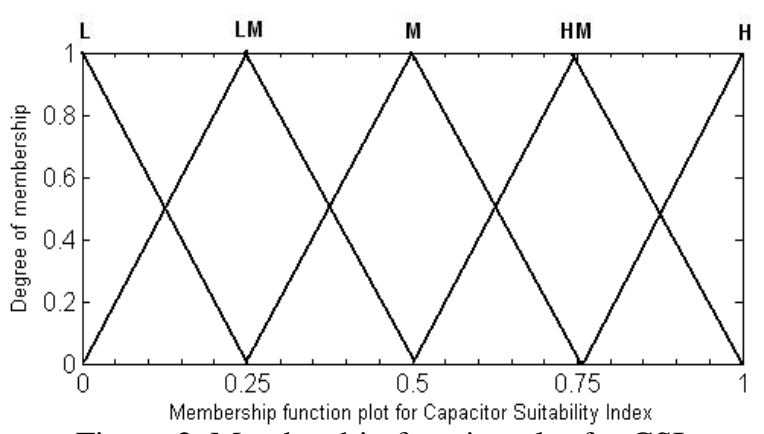

Figure 3. Membership function plot for CSI

Rules framed in the form "IF premise (or antecedent) THEN conclusion (or consequent)" are used to determine the suitability of a particular node or location for capacitor placement. The rules are framed and summarized in the fuzzy decision matrix as given in Table 1.

Table 1. Decision Matrix for determining the optimal capacitor locations

\begin{tabular}{|c|c|c|c|c|c|c|}
\hline \multicolumn{2}{|c|}{ AND } & \multicolumn{5}{c|}{ Per Unit Voltage } \\
\cline { 3 - 8 } & L & LN & N & HN & HH \\
\hline \multirow{3}{*}{ P } & L & LM & LM & L & L & L \\
\cline { 2 - 7 } & LM & M & LM & LM & L & L \\
\cline { 2 - 7 } & M & HM & M & LM & L & L \\
\cline { 2 - 7 } I & HM & HM & HM & M & LM & L \\
\cline { 2 - 7 } & H & H & HM & M & LM & LM \\
\hline
\end{tabular}

After receiving the inputs PLI and per unit voltage, the FIS, based on the rules framed in the decision matrix, calculates the suitability membership function of each node. This is then defuzzified in order to determine the node suitability ranking. The node having the highest value of CSI is most suitable for capacitor placement. Therefore the algorithm for finding the optimal capacitor location can be given as:

Step 1: Read the line and load data of the distribution system.

Step 2: Conduct load flow to determine the total active and reactive power losses.

Step 3: Conduct load flow to determine the total power losses after compensating reactive power at every node.

Step 4: Calculate the power loss reduction and PLI.

Step 5: PLI and per unit voltages are given as inputs to FIS

Step 6: Determine CSI by defuzzifying the outputs of FIS.

Step 7: the nodes having the highest value of CSI are most suitable for capacitor placement

\subsection{Introduction}

\section{BAT ALGORITHM}

Bat Algorithm (BA) is a nature inspired metaheuristic algorithm developed by Xin-She Yang in 2010. This paper presents the application of BA for finding the optimum sizes of capacitors after the optimum locations are determined using fuzzy logic.

Metaheuristic algorithms use certain trade-off of randomization and local search. Randomization provides a good way to move away from local search to the search on the global scale. Therefore, almost all the metaheuristic algorithms intend to be suitable for global optimization [18]. This algorithm is based on the echolocation behavior of micro bats [19]. Micro bats use a type of sonar, called, echolocation, to detect prey, avoid obstacles and locate their roosting crevices in the dark. These bats emit a very loud sound pulse and listen for the echo that bounces back from surrounding objects.

Bat algorithm is developed by idealizing some of the characteristics of micro bats. The approximated or idealized rules are:

a) All bats use echolocation to sense distance and they also know the difference between prey and barriers.

b) Bats fly randomly with velocity $v_{i}$ at position $x_{i}$ with a fixed frequency $f_{\min }$, varying wavelength $\lambda$ and loudness $\mathrm{A}_{0}$ to search for prey. They can automatically adjust the wavelength (or frequency) of their emitted pulses and the rate of pulse emission $\mathrm{r} \in[0,1]$ depending on the proximity of the target

c) Loudness varies from a large positive $\mathrm{A}_{0}$ to a minimum constant value $\mathrm{A}_{\min }$.

\subsection{Population}

The initial population i.e., number of virtual bats for BA (n) is generated randomly. The number of bats can be anywhere between 10 and 40 . After finding the initial fitness of the population for given objective function, the values are updated based on movement, loudness and pulse rate. 


\subsection{Movement of Virtual Bats}

The rules for updating the positions $\mathrm{x}_{\mathrm{i}}$ and velocities $\mathrm{v}_{\mathrm{i}}$ of the virtual bats are given as

where $\beta \in[0,1]$ is a random vector and

$$
\begin{aligned}
& \mathrm{f}_{\mathrm{i}}=\mathrm{f}_{\text {min }}+\left(\mathrm{f}_{\text {max }}-\mathrm{f}_{\text {min }}\right) \beta \\
& v_{i}^{t}=v_{i}^{t-1}+\left(x_{i}^{t}-\mathrm{x}_{*}\right) \mathrm{f}_{\mathrm{i}} \\
& x_{i}^{t}=x_{i}^{t-1}+v_{i}^{t}
\end{aligned}
$$

$\mathrm{X}_{*}$ is current global best solution.

Once a solution is selected among current best solutions, a new solution for each bat is generated locally using random walk

$\mathrm{x}_{\text {new }}=\mathrm{x}_{\mathrm{old}}+\varepsilon \mathrm{A}^{\mathrm{t}}$

where $\varepsilon \epsilon[-1,1]$ is a random number.

\subsection{Loudness and Pulse Emission}

The loudness $A_{i}$ and the rate of pulse emission $r_{i}$ are updated accordingly as the iterations proceed. The loudness decreases and rate of pulse emission increases as the bat closes on its prey i.e., the equations for convergence can be taken as

where $\alpha$ and $\gamma$ are constants.

$$
\begin{aligned}
& A_{i}^{t+1}=\alpha A_{i}^{t} \\
& r_{i}^{t+1}=r_{i}^{0}[1-\exp (-\gamma \mathrm{t})]
\end{aligned}
$$

For any $0<\alpha<1$ and $\gamma>0$, we have

$$
A_{i}^{t} \rightarrow 0, \quad r_{i}^{t} \rightarrow r_{i}^{0} \quad \text { as } \mathrm{t} \rightarrow \infty
$$

The initial loudness $\mathrm{A}_{0}$ can typically be $[1,2]$, while the initial emission rate $r_{i}^{0}$ can be $[0,1]$.

\subsection{Implementation of $\mathrm{BA}$}

From the equations above, we can implement BA in following steps:

Step 1: Initialize number of bats, number of iterations, loudness $A_{0}$, and pulse rate $r_{i}$.

Step 2: Generate the population randomly.

Step 3: Perform load flow to determine active power loss for generated population.

Step 4: Select minimum value as current best solution.

Step 5: Update frequency, velocity and position of bats using equations (2) to (5).

Step 6: Perform load flow for updated values and determine the losses.

Step 7: If the obtained losses are less than the current best solution, then replace the current best solution with the present obtained value and update loudness and pulse rate using equations (6) and (7).

Step 8: Repeat steps 5 to 7 until the given number of iterations are completed.

\section{RESULTS}

The optimal capacitor placement using Fuzzy and BA was applied on IEEE-15 and IEEE-34 bus systems and the results obtained are very encouraging. The method places capacitors at less number of locations with optimum sizes and offers much saving in investment and maintenance.

The proposed method has been programmed using MATLAB 7.12 and run on an Intel core i5 personal computer. The various parameters used in the algorithm are $n=40, \mathrm{~A}_{0}=1, \mathrm{r}_{0}=0.1, \alpha=0.9$ and $\gamma=0.1$. The losses (in $\mathrm{kW}$ ) before and after compensation are tabulated below:

Table 2. Test results of 15 and 34 bus systems

\begin{tabular}{|c|c|c|}
\hline & $\begin{array}{c}\text { Before } \\
\text { Compensation }\end{array}$ & $\begin{array}{c}\text { After } \\
\text { Compensation }\end{array}$ \\
\hline 15 bus & 61.7993 & 29.0686 \\
\hline 34 bus & 221.72 & 158.5951 \\
\hline
\end{tabular}

The results show that $53.05 \%$ reduction in power losses for 15 bus system and $28.47 \%$ reduction in power losses for 34 bus system are obtained when compared to uncompensated line. 


\section{CONCLUSIONS}

This paper has presented a novel method to determine the optimum capacitor locations using fuzzy approach and capacitor sizing problem for loss minimisation using BA method. The proposed method has been tested with several bus systems and the results are encouraging. Compared to the conventional methods, the FIS considers loss reduction and voltage profile simultaneously to decide the most suitable nodes for capacitor placement. By installing capacitors at all potential locations, the real power loss has been reduced significantly. BA iteratively searches the optimal capacitor sizes effectively for maximum loss reduction. The parameters $\mathrm{A}_{0}$, $\mathrm{r}_{0}, \alpha, \gamma$ play an important role in the performance of BA. The convergence depends highly on the parameters mentioned above.

\section{REFERENCES}

[1] Haque M.H., "Capacitor placement in radial distribution systems for loss reduction", IEEE Proceedings on Generation, Transmission and Distribution, vol. 146, no-5, pp 501-505, September 1999.

[2] M.E. Baran and F.F. Wu, "Optimal capacitor placement on radial distribution systems", IEEE Trans. Power Delivery, vol. 4, pp.725-734, Jan.1989.

[3] M.E. Baran and F.F. Wu, "Optimal sizing of capacitors placed on radial distribution systems", IEEE Trans. Power Delivery, vol. 4, pp.735-743. Jan.1989.

[4] S.Civanlar, J.J.Grainger, "Volt/Var control on distribution systems with lateral branches using shunt capacitors and voltage regulators, Part III: The numerical results", IEEE Trans. Power Apparatus and Systems, vol.4, pp. 32913297, Nov. 1985.

[5] M.Ponnavaiko, K.S.Prakasa Rao, "Optimal choice of fixed and switched capacitors on radial distribution feeders by the method of local variations", IEEE Trans. Power Apparatus and Systems, vol.-102, pp.1607-1615, Jun.1983

[6] M.Chis, M.M.A.Salama, S.Jayaram, "Capacitor placement in distribution systems using heuristic search strategies", IEEE Proceedings generation, Transmission, Distribution, vol.144, pp. 225-230, May. 1997.

[7] M.A.Salma, A.Y.Chikhani, "A simplified network approach to the VAR control problem for distribution systems", IEEE Trans. Power Delivery, vol.8, pp.1529-1535, Jul. 1993.

[8] H.D.Chiang, J.C.Wang, O.Cockings, H.D.Shin, "Optimal capacitor placements in distribution systems: Part I, Part II", IEEE Trans. Power Delivery, vol.5, pp 634- 649, Apr. 1990.

[9] Sundhararajan S. and Pahwa A., "Optimal selection of capacitors for radial distribution systems using a genetic algorithm”, IEEE Transactions on Power Systems, vo1.9, no.3, pp. 1499-1507, August 1994.

[10] B.Das, P.K.Varma, "Artificial neural network based optimal capacitor switching in a distribution system", Electric Power Systems Research, vol.60, pp.55-62, 2001.

[11] M.S.Calovic, A.T.Saric, "An integrated fuzzy for multi objective capacitor placement optimization in distribution networks", Electric Power Components and Systems, vol.30, pp.947-966, 2002.

[12] Prakash K. and Sydulu M., "Particle swarm optimization based capacitor placement on radial distribution systems", IEEE Power Engineering Society general meeting 2007, pp. 1-5, June 2007.

[13] P. V. Prasad, S. Sivanagaraju and N. Sreenivasulu, "A fuzzy-genetic algorithm for optimal capacitor placement in radial distribution systems", APRN Journal of Engineering and Applied Sciences, vol.2, no.3, pp.28-32, June 2007.

[14] Jianwu Li and Yao Lu, "An efficient real coded genetic algorithm for numerical optimization problems", IEEE Third International Conference on Natural Computation ICNC-2007.

[15] M. Damodar Reddy, V. C. Veera Reddy, "Optimal Capacitor Placement Using Fuzzy And Real Coded Genetic Algorithm For Maximum Savings", Journal of Theoretical and Applied Information Technology,

[16] Usha Reddy V, Dinakara Prasad Reddy P, "Differential Evolution Method for Capacitor Placement of Distribution Systems", International Journal of Advanced Engineering Sciences and Technologies, vol.4, no.2, pp. 23-28, 2011.

[17] Das D., Kothari D.P. and Kalam A., "Simple and efficient method for load flow solution of radial distribution networks", Electrical Power \& Energy Systems, vol. 17, no. 5, pp.335-346, 1995.

[18] X. S. Yang, Nature-Inspired Metaheuristic Algorithms (Luniver Press, 2008).

[19] X. S. Yang, "A New Metaheuristic Bat-Inspired Algorithm", Nature Inspired Cooperative Strategies for Optimisation (NISCO 2010), Studies in Computational Intelligence, Springer Berlin, 284, Springer, 66-74. 